\title{
CIRURGIA ESTEREOTÁXICA GUIADA PARA ANGIOMAS CAVERNOSOS
}

\author{
MURILO S. MENESES*, VANESSA C. DALLOLMO**, CHARLES KONDAGESKI***, \\ RICARDO RAMINA****, SONIVAL HUNHEVICZ****, ARI A. PEDROZO****
}

\begin{abstract}
RESUMO - Os angiomas cavernosos intracerebrais são lesões que podem causar hemorragias, crises convulsivas e déficits neurológicos. Essa patologia passou a ter diagnóstico mais precoce com o advento da ressonância magnética. A remoção radical por cirurgia é o melhor método de tratamento. Devido a frequente localização subcortical ou profunda, a utilização de métodos de localização por imagem como a estereotaxia apresentam várias vantagens como: menor incisão de pele, craniotomia e manipulação do tecido cerebral, consequentemente com menor morbidade. Apresentamos uma série de nove angiomas cavernosos tratados por ressecção cirúrgica radical guiada por estereotaxia. Em todos os casos o diagnóstico foi realizado por ressonância magnética e houve confirmação anátomo-patológica. A média de idade dos pacientes foi 30 anos, variando entre 20 e 54 anos. Como morbidade transitória, um paciente teve uma crise convulsiva no $3^{\circ}$ dia pós-operatório e uma paciente apresentou disfasia e hemiparesia no $2^{\circ}$ dia pós-operatório, com recuperação total na evolução. No total, a remoção foi radical em todos os casos e os pacientes permanecem sem nenhum déficit neurológico relacionado à cirurgia.
\end{abstract}

PALAVRAS-CHAVE: angioma cavernoso, estereotaxia, microcirurgia.

\section{Stereotactic-guided surgery for cavernous angioma}

ABSTRACT - Intracerebral cavernous angiomas may cause hemorrhage, epileptic seizures and neurological deficits. The diagnosis of these lesions became easier with the advent of the magnetic resonance image (MRI). Radical resection is the treatment of choice. Due to frequent subcortical or deep location, image-guided techniques, such as stereotactic-guided surgery, offer many advantages as smaller skin incision and craniotomy, less brain manipulation with consequently lower morbidity. We present a series of nine cavernous angiomas treated by stereotactic-guided radical surgical resection. The diagnosis was done by MRI and confirmed by pathologic studies in all cases. Mean age of patients was 30 years old (range 20-54 years). Postoperative morbidity occurred in two cases: one patient had a convulsion on the third postoperative day and the other presented dysphasia and hemiparesis on the second postoperative day, both with total recovery. Total resection of the lesion was possible in all cases with no neurological deficit.

KEY WORDS: cavernous angioma, stereotaxis, microsurgery.

Angiomas cavernosos são malformações vasculares compostas principalmente de aglomerados de vasos de paredes finas, sem vasos arteriais nutridores importantes e nenhum tecido nervoso intersticial $^{1}$. Essas malformações estão quase sempre associadas a gliose reativa e a depósitos de hemossiderina circundando a lesão ${ }^{2}$, podendo ser extensas e apresentar calcificações ${ }^{3}$. Segundo diferentes estudos, os angiomas cavernosos representam $10 \%$ das malformações vasculares cerebrais e estão presentes em 0,4 a $0,8 \%$ da população ${ }^{4}$.

Cerca de $10 \%$ dos angiomas são múltiplos e $5 \%$ têm características familiares, podendo nestes casos apresentar padrão genético autossômico dominante 5 . Embora as formas familiares e esporádicas

Serviço de Neurocirurgia, Instituto de Neurologia de Curitiba, PR. *Professor de Anatomia da Universidade Federal do Paraná (UFPR) e Neurocirurgião do Instituto de Neurologia de Curitiba (INC); **Estudante de Medicina, UFPR; ***Médico Residente de Neurocirurgia do INC; ****Neurocirurgião do INC.

Dr. Murilo S. Menezes - Avenida Getúlio Vargas 2159 - 80250-180 Curitiba PR - Brasil. 
dos angiomas cavernosos pareçam ser similares clinicamente, uma diferença importante consiste na grande frequência de múltiplas lesões nos pacientes de herança familiar. Em séries abrangendo somente casos familiares, $84 \%$ dos casos apresentam mais de um angioma. Essa porcentagem é bastante significativa se comparada com o índice de $25 \%$ de lesões múltiplas em pacientes sem história familiar ${ }^{4}$.

Os angiomas cavernosos passaram a ser diagnosticados mais facilmente com o advento da ressonância magnética (RM), pois a tomografia computadorizada (TC) geralmente só evidencia a lesão após sangramentos ${ }^{2}$. O diagnóstico é realizado após hemorragias, crises convulsivas e déficits neurológicos. A apresentação clínica dessas malformações é de epilepsia em $40 \%$ a $60 \%$ dos casos, de déficits neurológicos em $30 \%$ e de cefaléia em $25 \%$.

Existe índice elevado de sangramento na evolução natural dos angiomas cavernosos, variando em alguns estudos de $56 \%^{7}$ a $79 \%{ }^{8}$. Esses sangramentos podem causar déficits neurológicos e até óbito. Após a primeira hemorragia, os angiomas cavernosos tendem a apresentar ressangramentos mais frequentes e mais graves?.

A remoção cirúrgica é necessária para evitar as complicações da evolução natural dessas malformações. Devido ao fato de frequentemente apresentarem localização subcortical e profunda e o tamanho da lesão ser relativamente pequeno, a remoção cirúrgica pode ser extremamente facilitada pelo uso de um sistema guiado por imagem ${ }^{6,10}$. A cirurgia estereotáxica permite a ressecção radical da malformação de forma muito precisa, por um reduzido acesso e com menor agressão ao tecido cerebral, resultando em menor morbidade.

O uso da radiocirurgia ainda é bastante controverso e reservado somente aos pacientes que não podem ser submetidos a cirurgia. As dosagens corretas de irradiação ainda não estão bem estabelecidas ${ }^{2}$.

Apresentamos neste estudo uma série de nove angiomas cavernosos intracerebrais removidos por cirurgia estereotáxica guiada.

\section{MÉTODO}

Entre 1992 e 1999, 37 casos de angiomas cavernosos foram operados no Serviço de Neurocirurgia do Hospital das Nações de Curitiba. No mesmo período, entre 355 cirurgias estereotáxicas, 36 casos de lesões intracerebrais foram tratados por cirurgia guiada. Uma série de 9 casos de angiomas cavernosos removidos de forma radical por cirurgia estereotáxica guiada foi estudada e é apresentada neste trabalho.

A idade média dos pacientes foi 30 anos, variando de 20 a 54 anos. Seis pacientes eram do sexo feminino e 3 do sexo masculino. Quatro pacientes apresentaram cefaléia como sintoma principal, outros 4 pacientes tiveram crises convulsivas e 1 teve déficit neurológico e crise convulsiva. A localização dos angiomas cavernosos foi temporal em 3 casos, parietal em 3, frontal em 2, e parieto-occipital em 1 caso. Cinco apresentaram sinais de hemorragia nos exames de imagem.

O diagnóstico por imagem foi realizado por RM em todos os casos e outros exames, como TC e angiografia cerebral, foram utilizados para alguns pacientes.

\section{RESULTADOS}

A técnica cirúrgica utilizada foi a mesma em todos os casos. O marco estereotáxico (Fig 1) é fixado ao crânio do paciente sob anestesia local e sedação com diprivan (Propofol). Um exame de RM (Fig 2-A) permite a determinação das coordenadas estereotáxicas do angioma cavernoso. $\mathrm{O}$ paciente é conduzido ao centro cirúrgico e submetido a anestesia geral ou permanece sob anestesia local, quando a lesão se localiza em área eloquente. A incisão da pele e a craniotomia, de tamanho reduzido, são realizadas com orientação pelas coordenadas estereotáxicas e evitando-se áreas cerebrais altamente funcionais. A abertura da dura-máter (Fig 3-A) possibilita uma pequena corticotomia para o acesso microcirúrgico. O sistema estereotáxico guia o trajeto até a lesão, que é removida por técnicas microcirúrgicas convencionais (Figs 2-B e 3-B).

A ressecção foi total e houve confirmação anátomo-patológica em todos os casos. Os dados referentes aos pacientes são apresentados na Tabela 1. 
Seis pacientes permaneceram totalmente assintomáticos após a cirurgia e um manteve o mesmo déficit pré-operatório. Um paciente apresentou crise convulsiva no $3^{\circ}$ dia pósoperatório, permanecendo assintomático desde então. Uma paciente com lesão temporal posterior esquerda apresentou no $2^{\circ}$ dia pósoperatório disfasia e hemiparesia com recuperação total em 45 dias, retornando a sua atividade profissional de professora. Os 9 pacientes permanecem sem nenhum déficit neurológico relacionado à cirurgia.

\section{DISCUSSÃO}

Devido à frequente incidência de sangramento dos angiomas cavernosos, a melhor opção de tratamento é a remoção cirúrgica radical. Desta forma, o paciente fica livre dos riscos das complicações relacionadas às hemorragias, como déficits neurológicos ou mesmo óbito. Essas lesões representam causa frequente de epilepsia. Numa análise de 50 pacientes ${ }^{11}$, notou-se que aqueles com menos de 2 meses de epilepsia ou somente 1 crise

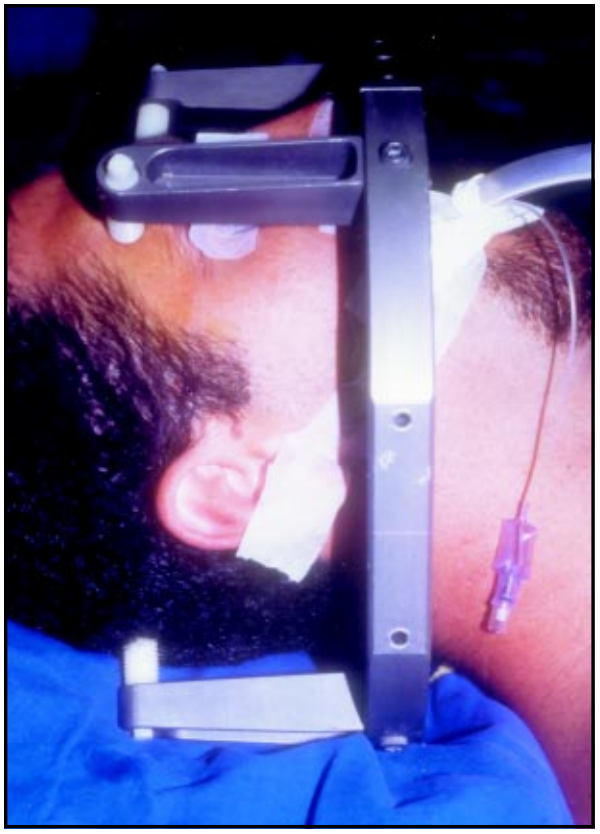

Fig 1. Marco estereotáxico fixado ao crânio do paciente. convulsiva antes da cirurgia, tiveram abolição total das crises (100\%) após a ressecção do angioma cavernoso. Entretanto os pacientes com convulsões há mais de 12 meses, ou com mais de 5 crises por mês, tiveram resultados de aproximadamente $50 \%$ de controle total da epilepsia. Essas observações são importantes no sentido de que a lesionectomia seja indicada precocemente.

Tabela 1. Dados referentes aos pacientes operados.

\begin{tabular}{cccccccc}
\hline Casos & Idade & Sexo & Clínica & Localização & Sangramento & Ressecção & Evolução \\
\hline 1 & 39 & Fem & Convulsão & Frontal D & Não & Total & Normal \\
2 & 22 & Fem & Cefaléia & Temporal E & Sim & Total & Normal \\
3 & 31 & Masc & Cefaléia & Parietal D & Não & Total & Normal \\
4 & 31 & Fem & Cefaléia & Parieto-occipital E & Sim & Total & Normal \\
5 & 20 & Fem & Convulsão & Parietal D & Sim & Total & Normal \\
6 & 28 & Fem & Cefaléia e & Temporal E & Sim & Total & Disfasia, \\
& & & Parestesias & & & hemiparesia, \\
7 & 26 & Masc & Convulsão & Frontal D & Sim & Total & Normal \\
8 & 26 & Masc & Convulsão & Temporal E & Não & Total & Convulsão, \\
& & & Femánotal & & assintomático \\
9 & 54 & Fem & Paresia E, & Parietal D & Não & Total & Normal \\
& & & Convulsão & & & inalterado, \\
& & & & & sem convulsão \\
\hline
\end{tabular}

D, direita; E, esquerda. 


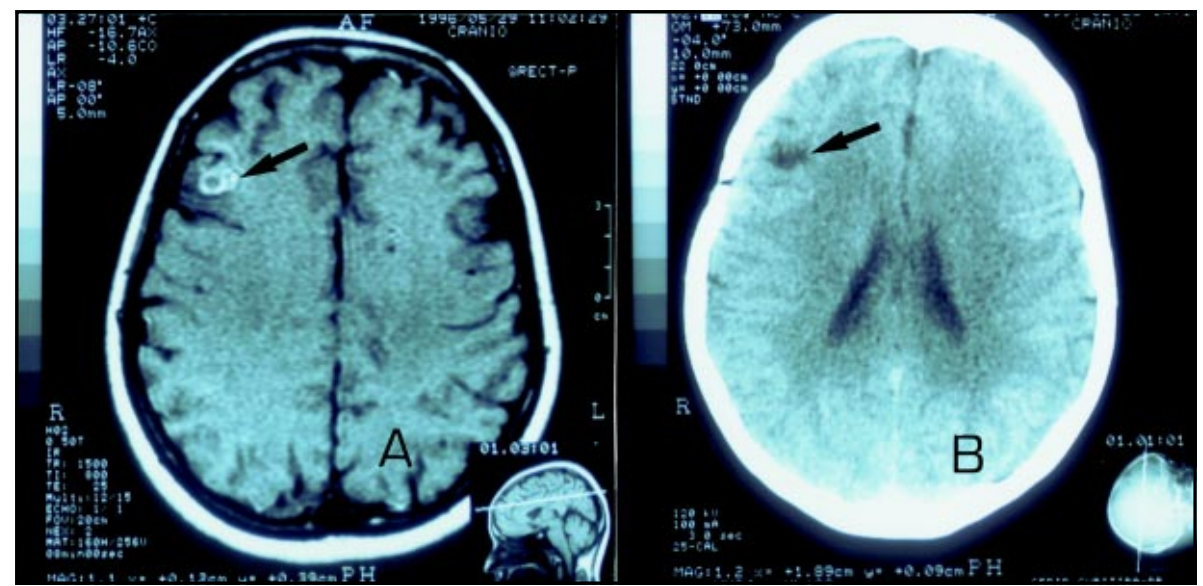

Fig 2. A, exame de ressonância magnética, sequência ponderada em T1, em corte axial, mostrando angioma cavernoso frontal direito (flecha); B, exame pós-operatório de tomografia computadorizada em corte axial, mostrando a remoção radical da lesão (flecha).

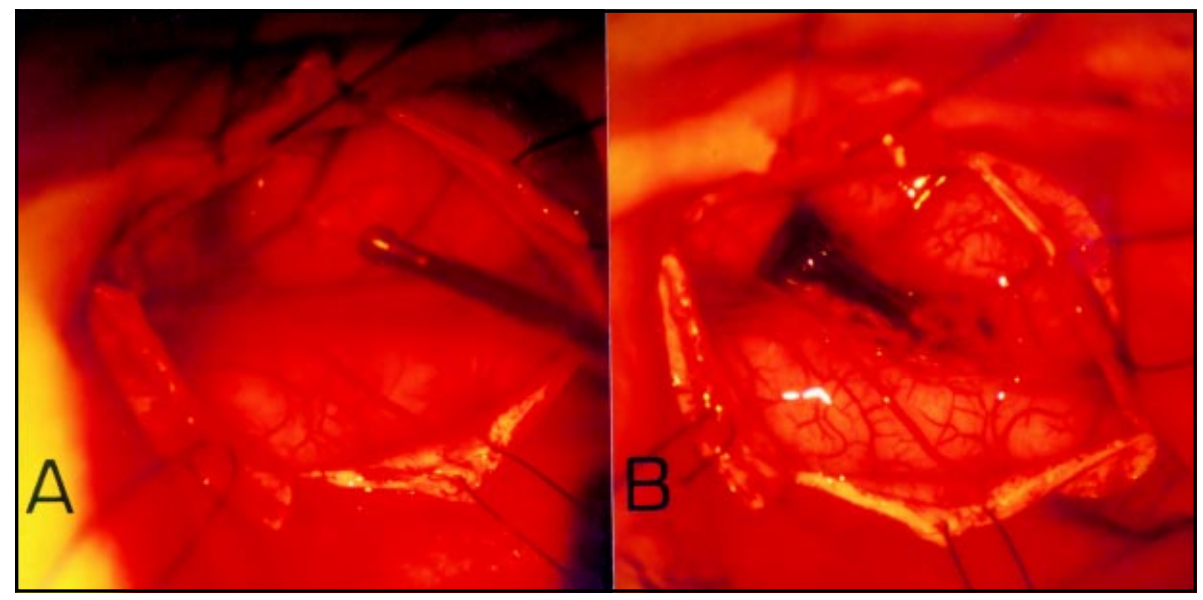

Fig 3. A, visão da superfície cerebral com determinação da localização estereotáxica do angioma cavernoso; $B$, visão da mesma área após a remoção microcirúrgica da lesão.

Em dois casos da nossa série, após uma evolução pós-operatória normal, apareceram sintomas como crise convulsiva e disfasia. Nossa explicação para o fato é que, por se tratar de uma lesão vascular, existe a possibilidade de ocorrer edema cerebral tardio. $\mathrm{O}$ uso mais prolongada de antiedematosos poderia evitar alterações neurológicas transitórias ou mesmo definitivas. Esses dois pacientes apresentaram controle total das alterações neurológicas na evolução.

Os angiomas cavernosos são lesões com bom plano de clivagem e que permitem ressecção total na grande maioria dos casos. Frequentemente localizam-se em áreas subcorticais ou profundas. A cirurgia convencional, sem sistema de orientação por imagem, é menos precisa. Os acessos utilizados são maiores, mais agressivos, com exposição cirúrgica desnecessária e maior manipulação de tecido nervoso, podendo provocar maior morbidade e mortalidade. A estereotaxia é método simples que permite a localização da lesão com precisão da ordem de $1 \mathrm{~mm}^{10}$. A fixação do marco estereotáxico no crânio do paciente é semelhante à utilizada com o segurador de cabeça de Mayfield, empregada nas neurocirurgias de rotina, não representando qualquer dificuldade adicional. Os sistemas 
estereotáxicos modernos, sendo isocêntricos, possibilitam que uma lesão cerebral profunda seja abordada por diferentes vias com tamanho reduzido, evitando-se áreas corticais eloquentes.

Angiomas venosos estão frequentemente associados a angiomas cavernosos. Não há indicação cirúrgica de angioma venoso, pois sua remoção pode resultar em infarto venoso grave ${ }^{12}$.

Nessa série de 9 pacientes, a ressecção do angioma cavernoso foi total em todos os casos. Não houve qualquer déficit neurológico no período pós-operatório imediato. Os dois pacientes que apresentaram alterações no segundo dia pós-operatório tiveram recuperação total, permanecendo assintomáticos. Essa alteração tardia, possivelmente, está relacionada a edema vascular.

A localização estereotáxica, realizada sob anestesia local no centro de neuroimagem, é rápida e permite uma cirurgia mais segura. A incisão de pele, a craniotomia e a manipulação de tecido cerebral são menores, e o tempo de ressecção do angioma é mais curto devido à facilidade de localização da lesão. Desta forma, consideramos a cirurgia estereotáxica guiada indicada para ressecção total dos angiomas cavernosos cerebrais subcorticais e profundos.

\section{REFERÊNCIAS}

1. Adams RB, Victor M. Principles of neurology. 5 th Ed. New York: McGraw Hill, 1993:560-561.

2. Rocco CD, Iannelli A, Tamburrini G. Cavernomas of the central nervous system in children. Acta Neurochir (Wien) 1996;138:1267-1274.

3. Ramina R, Ingunza W, Vonofakos D. Cystic cerebral cavernous angioma with dense calcification. J Neurosurg 1980;52:259262.

4. Moriarity JL, Wetzel M, Clatterbuck RE et al. The natural history of cavernous malformations: a prospective study of 68 patients. Neurosurgery 1999;44:1166-1174.

5. Marchuk DA, Gallione CJ, Morrisson LA, et al. A locus for cerebral cavernous malformations maps to chromosome $7 \mathrm{q}$ in two families. Genomics $1995 ; 28: 31$.

6. Zevgaridis D, Van Velthoven V, Ebeling U, Reulen HJ. Seizure control following surgery in supratentorial cavernous malformations: a retrospective study in 77patients. Acta-Neurochir (Wien) 1996;138:672-677.

7. Aiba T, Tanaka R, Koike T, et al. Natural history of intracranial cavernous malformations. J Neurosurg 1995;83:56-59.

8. Scott RM, Barnes P, Kupsky W, Adelman LS. Cavernous angiomas of the central nervous system in children. J Neurosurg 1992;76:38-46.

9. Duffau H, Capelle L, Sichez JP, et al. Early radiologically proven rebleeding from intracranial cavernous angiomas: report of cases and review of the literature. Acta Neurochir (Wien) 1997;139:914-922.

10. Meneses MS, Huss HHA, Coelho MN, et al. Cirurgia estereotáctica para processos expansivos intracranianos: diagnóstico e tratamento. Arq Neuropsiquiatr 1996;54:64-70.

11. Rossi GF. Epileptogenic cavernous angiomas: the role of lesionectomy. Crit-Rev Neurosurg 1997;7:52-57.

12. Vishteh AG, Sankhla S, Anson JA, Zabranski JM, Spetzler RF. Surgical resection of intramedullary spinal cord cavernous malformations: delayed complications, long-term outcomes, and association with cryptic venous malformations. Neurosurgery 1997;41:1094-1101. 